\title{
On a conjecture of Kontsevich and variants of Castelnuovo's lemma
}

\author{
J. M. LANDSBERG \\ Laboratoire de Mathématiques, Université Paul Sabatier, UFR-MIG 31062 Toulouse Cedex 4, \\ France; e-mail: jml@picard.ups-tlse.fr
}

Received 19 February 1997; accepted in final form 1 October 1997

\begin{abstract}
Let $A=\left(a_{j}^{i}\right)$ be an orthogonal matrix (over $\mathbb{R}$ or $\mathbb{C}$ ) with no entries zero. Let $B=\left(b_{j}^{i}\right)$ be the matrix defined by $b_{j}^{i}=1 / a_{j}^{i}$. M. Kontsevich conjectured that the rank of $B$ is never equal to three. We interpret this conjecture geometrically and prove it. The geometric statement can be understood as variants of the Castelnuovo lemma and Brianchon's theorem.
\end{abstract}

Mathematics Subject Classifications (1991): 114E07, 14M210, 115A99.

Key words: rational curves, Cremona transform, quadrics, Castelnuovo's lemma, Brianchon's theorem, Gale transform, association.

\section{Definitions and statements}

DEFINITION 1.1. Given a $k \times l$ matrix $A=\left(a_{\alpha}^{i}\right), 1 \leqslant i \leqslant k, 1 \leqslant \alpha \leqslant l$, with no entries zero, define the Hadamard inverse of $A, B=\left(b_{\alpha}^{i}\right)$, by $b_{\alpha}^{i}=1 / a_{\alpha}^{i}$. (The name is in analogy with the Hadamard product.)

Maxim Kontsevich conjectured the following:

CONJECTURE 1.2 (Kontsevich (1988)). Let $A$ be an orthogonal matrix (over $\mathbb{R}$ or $\mathbb{C})$ with no entries zero. Let $B$ be the Hadamard inverse of $A$. Then the rank of $B$ is never equal to three.

At first glance, (1.2) may not appear all that striking because based on a naive count, one would not expect any low rank Hadamard inverses of orthogonal matrices to exist (see (1.16)). However, Kontsevich asserted and we show the following:

THEOREM 1.3. The space of $m \times m$ orthogonal matrices with rank two Hadamard inverses is $(2 m-3)$-dimensional.

We will rephrase (1.2), (1.3) in geometric language and prove them. First we will need some definitions:

DEFINITION 1.4. Let $V=\mathbb{C}^{n+1}$ or $\mathbb{R}^{n+1}$, let $Q \in S^{2} V^{*}$ be a nondegenerate quadratic form. Two points $z, w \in \mathbb{P} V$ are said to be polar, or more precisely, 
$Q$-polar if the corresponding lines in $V$ are $Q$-orthogonal. Given a point $z \in \mathbb{P} V$, the polar hyperplane of $z$ with respect to $Q, H_{Q, z}$, is the hyperplane of $Q$-polar points to $z$. A set of points $z_{0}, \ldots, z_{n}$, none lying on the quadric in $\mathbb{P} V$ defined by $Q$, is said to be apolar with respect to $Q$ if they are all mutually polar. (In particular, vectors in $V$ representing them give a $Q$-orthogonal basis of $V$.) A quadratic form $P$ is said to be apolar to $Q$ if $\operatorname{trace}_{Q} P=0$. ( $\operatorname{trace}_{Q} P$ is defined by considering the induced maps $P^{\prime}: V \rightarrow V^{*}, Q^{\prime \prime}: V^{*} \rightarrow V ; \operatorname{trace}_{Q} P:=\operatorname{trace}\left(Q^{\prime \prime} \circ P^{\prime}\right)$.) or $\mathbb{C}$.

In what follows, unless we specify the ground field, it may be taken to be $\mathbb{R}$

DEFINITION 1.5. Given a set of points $z_{0}, \ldots, z_{n}$ spanning $\mathbb{P}^{n}$, define the standard Cremona transform of $\mathbb{P}^{n}$ with respect to $\left\{z_{i}\right\}$ to be the rational map $\phi: \mathbb{P}^{n} \rightarrow \mathbb{P}^{n}$, obtained by first blowing up the codimension two spaces spanned by subsets of $(n-1)$-tuples of the points, and then blowing down the $n$ hyperplanes containing sets of $n$ of the points. In coordinates, if

$$
z_{i}=[0, \ldots, 0,1,0, \ldots, 0],
$$

where the 1 occurs in the $i$ th slot, the map is

$$
\phi\left(\left[x^{0}, \ldots, x^{n}\right]\right)=\left[\frac{1}{x^{0}}, \ldots, \frac{1}{x^{n}}\right] .
$$

Note that the images of the blown down hyperplanes determine a coordinate simplex in the image $\mathbb{P}^{n}$ which we will call the image simplex.

Conjecture (1.2) is equivalent to:

THEOREM 1.8 (Version 1). Let $z_{0}, \ldots, z_{n}$ and $p_{0}, \ldots, p_{n}$ be two sets of points spanning $\mathbb{P}^{n}$, each set apolar with respect to a nondegenerate quadratic form $Q$. Let $\phi$ denote the Cremona transform defined by the $\left\{z_{i}\right\}$. If the points $\phi\left(p_{i}\right)$ fail to span a $\mathbb{P}^{3}$, then they span exactly a $\mathbb{P}^{1}$.

THEOREM 1.8 (Version 2). Let $z_{0}, \ldots, z_{n}$ and $p_{0}, \ldots, p_{n}$ be two sets of points spanning $\mathbb{P}^{n}$, each set apolar with respect to a nondegenerate quadratic form $Q$. Let $\Sigma$ be the space of hypersurfaces of degree $n$ having multiplicities of order $n-2$ at each $z_{i}$. ( $\Sigma$ is a $\mathbb{P}^{n}$.) Let $\Lambda:=\left\{P \in \Sigma \mid p_{i} \in P \forall i\right\}$. If codim $\Lambda \leqslant 2$ then codim $\Lambda=1$. In this case the points $z_{i}, p_{i}$ all lie on a rational normal curve.

Equivalence of versions 1 and $2 . \Sigma$ is the space of inverse images under $\phi$ of the hyperplanes in $\mathbb{P}^{n}$, and for any $\mathbb{P}^{1} \subset \mathbb{P}^{n}, \phi^{-1}\left(\mathbb{P}^{1}\right)$ is a (possibly degenerate) rational normal curve. (The $\mathbb{P}^{1}$ 's that we will consider will yield non-degenerate rational normal curves.)

One form of the classical Brianchon theorem says that given a conic in the plane and given two triangles circumscribing the conic, then the six points consisting of 
the vertices of the two triangles all lie on another conic. The $n=3$ case of (1.8) provides a variant of this which was originally proven by Weddle and Zeuthen (see [EP2] for an exposition of their work).

COROLLARY 1.9 (Weddle, Zeuthen). Let $Q \subset \mathbb{P}^{2}$ be a smooth conic. Let $z_{0}, z_{1}, z_{2}$ and $p_{0}, p_{1}, p_{2}$ be two sets of $Q$-apolar points. Then the six points $z_{0}, z_{1}, z_{2}, p_{0}, p_{1}, p_{2}$ all lie on a conic.

Writing $p_{j}=\left[p_{j}^{0}, p_{j}^{1}, p_{j}^{2}\right], z_{i}$ as in (1.6), and let $Q$ have equation $\Sigma_{i}\left(x^{i}\right)^{2}=0$, the conic all six points lie on is

$$
\left(p_{0}^{2} p_{1}^{2} p_{2}^{2}\right) x^{0} x^{1}+\left(p_{0}^{1} p_{1}^{1} p_{2}^{1}\right) x^{0} x^{2}+\left(p_{0}^{0} p_{1}^{0} p_{2}^{0}\right) x^{1} x^{2} .
$$

More generally, we have:

PROPOSITION 1.11. Let $z_{0}, \ldots, z_{n}$ and $p_{0}, \ldots, p_{n}$ be two sets of points spanning $\mathbb{C P}^{n}=\mathbb{P} V$, each set apolar with respect to a smooth quadric $Q$. Let $\mathcal{Q}=\{P \in$ $\left.\mathbb{P} S^{2} V^{*} \mid z_{i}, p_{i} \in P \forall i\right\}$. Then $\operatorname{dim} \mathcal{Q}=\left(\begin{array}{l}n \\ 2\end{array}\right)-1$.

Proof. The dimension of the space of quadrics containing any $2 n+1$ points is $\left(\begin{array}{l}n \\ 2\end{array}\right)-1$, so we need to show that any quadric containing all but possibly one of the points also contains the last point. Recall that for two quadratic forms $Q, P \in S^{2} V^{*}$, that $\operatorname{trace}_{Q} P=\Sigma_{i} P\left(v_{i}, v_{i}\right)$ is a well-defined number, where $\left\{v_{i}\right\}$ is any $Q$-orthonormal basis of $V$. Take $Q \in S^{2} V^{*}$ and let $\hat{p}_{i}$ and $\hat{z}_{i}$ be corresponding $Q$-orthonormal bases. We have

$$
P\left(\hat{z}_{0}, \hat{z}_{0}\right)+\cdots+P\left(\hat{z}_{n}, \hat{z}_{n}\right)=P\left(\hat{p}_{0}, \hat{p}_{0}\right)+\cdots+P\left(\hat{p}_{n}, \hat{p}_{n}\right) .
$$

If all the points but perhaps $p_{n}$ lie on $P$, we see that $p_{n}$ must as well.

Relation to Castelnuovo's Lemma 1.12. Castelnuovo's lemma says that if $2 n+3$ points lie on an $\left\{\left(\begin{array}{l}n \\ 2\end{array}\right)-1\right\}$-dimensional linear system of quadrics, then in fact they all lie on a rational normal curve. $\left\{\left(\begin{array}{l}n \\ 2\end{array}\right)-1\right\}$ is the dimension of the space of quadrics containing a rational normal curve and the point of Castelnuovo's lemma is that not only is the space of the correct dimension, but it is actually a space cutting out a rational normal curve. Here we only have $2 n+2$ points. (1.11) shows that the apolarity conditions imply that the $2 n+2$ points always lie on an $\left\{\left(\begin{array}{l}n \\ 2\end{array}\right)-1\right\}$-dimensional linear system of quadrics. When one adds the additional hypothesis on the Cremona images of the points, the system of quadrics cuts out a rational normal curve.

Proof of equivalence of (1.1) and (1.8). Write

$$
A=\left(p_{j}^{i}\right)
$$


and let

$$
p_{j}=\left[p_{j}^{0}, \ldots, p_{j}^{n}\right]
$$

Without loss of generality, take $z_{i}$ as in (1.6) and let $Q$ have equation $\Sigma_{i}\left(x^{i}\right)^{2}=0$. The Hadamard inverse of $A$ is given by the coordinates of the $\phi\left(p_{i}\right)$ up to the ambiguity of scales which do not effect rank, i.e. the rank of the Hadamard inverse of $A$ is the dimension of the span of the $\phi\left(p_{i}\right)$ plus one.

PROPOSITION 1.15 (Duality). Let $z_{0}, \ldots, z_{n}$ and $p_{0}, \ldots, p_{n}$ be two sets of points spanning $\mathbb{P}^{n}$, each set apolar with respect to a nondegenerate quadratic form $Q$. If the images of the $p_{i}$ under the Cremona transform defined by the $z_{i}$ lie on a $\mathbb{P}^{k}$, then images of the $z_{i}$ under the Cremona transform defined by the $p_{i}$ also lie on a $\mathbb{P}^{k}$.

(1.15) will follow from some remarks on the Gale transform given in Section 4.

PROPOSITION 1.16. There exist orthogonal rank $k$ Hadamard inverses for $m \times m$ complex matrices for all

$$
k \geqslant m-\sqrt{\frac{m^{2}}{2}+\frac{m}{2}-1} .
$$

Proof. Let $M=\mathbb{C}^{m} \otimes \mathbb{C}^{m}$ and let $\phi: \mathbb{P} M=\mathbb{P}^{m^{2}-1} \rightarrow \mathbb{P}^{m^{2}-1}$ be the Cremona transform defined by the standard coordinates. Write $n=m-1$.

Consider the Segre, $\operatorname{Seg}\left(\mathbb{P}^{n} \times \mathbb{P}^{n}\right)$, in $\phi(\mathbb{P} M)$ and let $\sigma_{k}\left(\operatorname{Seg}\left(\mathbb{P}^{n} \times \mathbb{P}^{n}\right)\right)$ denote its $k$ th secant variety, the closure of the union of all $\mathbb{P}^{k-1}$ 's spanned by $k$-ples of points of the Segre. Let $Y_{k}=\phi^{-1} \sigma_{k}\left(\operatorname{Seg}\left(\mathbb{P}^{n} \times \mathbb{P}^{n}\right)\right)$, so $Y_{k}$ is the space of matrices with Hadamard inverse of rank less than or equal to $k$. Note that $\operatorname{dim} Y_{k}=k(2 m-k)-1$.

Let $Z \subset \mathbb{P} M$ be the space of orthogonal columns, i.e.

$$
Z=\left\{A \mid A^{t} A \text { is diagonal }\right\} .
$$

$Z$ is a complete intersection of the $\left(\begin{array}{c}m \\ 2\end{array}\right)$ quadrics $\Sigma_{i} a_{j}^{i} a_{k}^{i}=0$ for all $j<k$. ( $Z$ is isomorphic to the variety of complete flags.) $Y_{k}$ will intersect $Z$ if

$$
k(2 m-k)-1+\left(\begin{array}{c}
m+1 \\
2
\end{array}\right)-1 \geqslant m^{2}-1,
$$

i.e., if

$$
k \geqslant m-\sqrt{\left(\begin{array}{c}
m+1 \\
2
\end{array}\right)-1} .
$$




\section{Proof of 1.8 .}

Let $A=\left(p_{j}^{i}\right)$ be as in (1.14) and let $\mathbb{P}^{n}=\phi\left(\mathbb{P}^{n}\right)$ have linear coordinates $y^{0}, \ldots, y^{n}$ adapted to the image simplex. Let $H_{i j k}(i<j<k)$ denote the hyperplane defined by the equation

$$
E_{i j k}=p_{i}^{0} p_{j}^{0} p_{k}^{0} y^{0}+\cdots+p_{i}^{n} p_{j}^{n} p_{k}^{n} y^{n} .
$$
that

Let $q_{j}=\phi\left(p_{j}\right)$ so $q_{j}^{i}=p_{j}^{0} \ldots \hat{p}_{j}^{i} \ldots p_{j}^{n}$, where the hat denotes omission. Note

$$
\operatorname{Span}\left\{q_{i}, q_{j}, q_{k}\right\} \subseteq H_{i j k}
$$

To verify (2.2), by symmetry it is sufficient to verify $q_{i} \in H_{i j k}$.

$$
\begin{aligned}
E_{i j k}\left(q_{i}\right) & =\Sigma_{l} p_{i}^{l} p_{j}^{l} p_{k}^{l}\left(p_{i}^{0} \ldots \hat{p}_{i}^{l} \ldots p_{i}^{n}\right) \\
& =\left(p_{i}^{0} \ldots p_{i}^{n}\right) \Sigma_{l} p_{j}^{l} p_{k}^{l} \\
& =\left(p_{i}^{0} \ldots p_{i}^{n}\right) \hat{Q}\left(\mathbf{p}_{j}, \mathbf{p}_{k}\right) \\
& =0
\end{aligned}
$$

where $\mathbf{p}_{j} \in V$ is a unit vector corresponding to $p_{j} \in \mathbb{P} V$.

Now say that the $q_{i}$ span a $\mathbb{P}^{2}$. Then all the $q_{l}$ 's are in the span of any $q_{i}, q_{j}, q_{k}$ spanning the $\mathbb{P}^{2}$. For the moment, assume we are in the case that there exist two points, say, $q_{0}, q_{1}$, such that no other $q_{i}$ lies on the line between $q_{0}$ and $q_{1}$. (This is always the case over $\mathbb{R}$, but over $\mathbb{C}$ there is the example of the nine flexes on a plane cubic.)

We claim that the intersection of $H_{012}, \ldots, H_{01 n}$ is at most a $\mathbb{P}^{1}$, which will prove (1.8) in this case.

To see the claim, say there were a linear relation among $E_{012}, E_{013}, \ldots, E_{01 n}$, e.g.

$$
a^{2} E_{012}+a^{3} E_{013}+\cdots+a^{n} E_{01 n}=0 .
$$

The coefficient of $y^{j}$ in (2.4) is

$$
a^{2} p_{0}^{j} p_{1}^{j} p_{2}^{j}+\cdots+a^{n} p_{0}^{j} p_{1}^{j} p_{n}^{j}=0 .
$$

This implies (since none of the $p_{j}^{i}$ are zero) that

$$
a^{2} p_{2}^{j}+\cdots+a^{n} p_{n}^{j}=0 \forall j
$$

i.e. that there is a linear relation among the columns of the matrix $A$ which is a contradiction. 
Now say there are three points, say $q_{0}, q_{1}, q_{2}$ that are colinear but no other $q_{j}$ lies on the line they span. We must show that among the hyperplanes containing the $\mathbb{P}^{2}$, that $(n-2)$ of them are independent. Say not, then for each $3 \leqslant \beta \leqslant n$ there must be a relation

$$
a_{3}^{\beta} E_{013}+\cdots+a_{n}^{\beta} E_{01 n}=a_{\beta} E_{02 \beta},
$$

i.e.,

$$
a_{3}^{\beta} p_{1}^{j} p_{3}^{j}+\cdots+a_{n}^{\beta} p_{1}^{j} p_{n}^{j}=a_{\beta} p_{2}^{j} p_{\beta}^{j} \forall j
$$

Note that if any of the $a_{\beta}$ are zero we are done by the above argument. Similarly there must be a relation among $H_{023}, \ldots, H_{02 n}$ and $H_{013}$ which implies an equation of the form:

$$
b_{3} p_{2}^{j} p_{3}^{j}+\cdots+b_{n} p_{2}^{j} p_{n}^{j}=b p_{1}^{j} p_{3}^{j} .
$$

Substituting the right-hand side of (2.8.3), . ., (2.8.n) into the left-hand side of (2.9) we obtain a relation involving $p_{1}^{j}$ in each term which divides out and we are left with a relation among the rows $p_{\beta}$ and thus a contradiction.

In the event, even more points are required, the same argument as above still works, only one must use more relations. (To our knowledge, there are no known configurations of points that span a plane with more than three points on each line.)

\section{Bases and the proof of (1.3)}

DEFINITION 3.1. A base $\Gamma$ is a set of $(n+2)$ points in $\mathbb{C P}^{n}$ in general linear position. The definition is motivated by the fact that all such $(n+2)$-ples of points are projectively equivalent. Note that there is an $(n-1)$-dimensional linear system of rational normal curves through $\Gamma$ which we will denote $\mathcal{R}_{\Gamma}$ and an $\left\{\left(\begin{array}{c}n+1 \\ 2\end{array}\right)-2\right\}$ dimensional linear system of quadric hypersurfaces through $\Gamma$, which we denote $\mathcal{Q}_{\Gamma}$.

The following is a slight modification of some facts in [Con] and [DO]:

LEMMA 3.2. If we fix a base $\Gamma=\left\{z_{0}, \ldots, z_{n}, p_{0}\right\}$ and a hyperplane $H$ with $\Gamma \cap H=\emptyset$, then there is a unique quadric $Q_{0}$ such that the $\left\{z_{i}\right\}$ are apolar with respect to $Q_{0}$ and $H$ is the polar hyperplane of $p_{0}$ with respect to $Q_{0}$.

Proof. Without loss of generality, take $z_{i}$ as vertex points as in (1.6) and take $p_{0}=[1, \ldots, 1]$. Say $H$ has equation $\Sigma_{i} a_{i} x^{i}=0$. All quadrics for which the $z_{i}$ are apolar are of the form $Q=\Sigma_{i} \lambda_{i}\left(x^{i}\right)^{2}$ for some constants $\lambda_{i}$. The $p_{0}$-polar hyperplane of such a quadric has the equation $\Sigma_{i} \lambda_{i} x^{i}$, so we must have $\lambda_{i}=a_{i}$, uniquely determining $Q_{0}$. 
LEMMA 3.3. If we fix a base $\Gamma=\left\{z_{0}, \ldots, z_{n}, p_{0}\right\}$, a hyperplane $H$ and $Q_{0}$ as above, then for all $R \in \mathcal{R}_{\Gamma}$, the set of $n+1$ points consisting of $R \cap H$ and $p_{0}$ is apolar with respect to $Q_{0}$.

Proof. See [DO] Lemma 5, p. 49.

Proof of (1.3). Fixing the $z_{i}$ as in (1.6) and $Q_{0}=\Sigma_{i}\left(x^{i}\right)^{2}$, i.e. fixing a copy of $S O(n+1)$, we are free to pick $p_{0}$ from an open set in $\mathbb{P}^{n}$, and then there is a $\mathbb{P}^{n-1}$,s worth of rational normal curves through $\left\{z_{i}, p_{0}\right\}$. We see that the dimension of complex orthogonal $(n+1) \times(n+1)$ matrices with rank-two Hadamard inverses is $2 n-1$. Finally, taking $Q_{0}$ as above over $\mathbb{R}$ the same count is still valid.

Note that a rank-one Hadamard inverse is impossible as $\phi$ is one to one off the hyperplanes that get blown down, and points on blown down hyperplanes correspond to a column vector with at least one entry equal to zero. (In fact the number of zeros in a column is the number of such hyperplanes the corresponding point lies on.)

\section{Some remarks on the Gale transform}

Everything in this section with the exception of Version 3 of Theorem 1.8 is classical and explained in modern language and greater generality in [DO], [EP1], and [EP2]. For our purposes, points will be distinct and in a sufficiently general linear position so that there is no need to be concerned with degenerate cases, and this will enable a simplified exposition.

DEFINITION 4.1. Let $\Gamma$ be a set of $r+s+2$ points in $\mathbb{P}^{r}$. A set $\Gamma^{\prime}$ of $r+s+2$ points in $\mathbb{P}^{s}$ is said to be associated to $\Gamma$ if when one chooses coordinates in the respective projective spaces and writes the points of $\Gamma$ as the rows of an $(r+1) \times(r+s+2)$ matrix $A$ and the points of $\Gamma^{\prime}$ as the rows of an $(s+1) \times(r+s+2)$ matrix $B$, that there exists a $(r+s+2) \times(r+s+2)$ diagonal matrix $\Lambda$ with a nonzero determinant such that $A \Lambda^{t} B=0$. If the point sets are sufficiently nice, there is a unique associated point set. (Of course, all this defined up to $P G L(r)$ and $P G L(s)$ actions).

The set $\Gamma^{\prime}$ is called the Gale transform of $\Gamma$. We will explain association in coordinates.

Let $0 \leqslant i, j \leqslant r$, and $0 \leqslant \alpha, \beta \leqslant s$. Write $\Gamma=\left\{z_{i}, p_{\alpha}\right\}$, and without loss of generality (assuming the $z_{i}$ are in general linear position) write $z_{i}=$ $[0, \ldots, 0,1,0, \ldots, 0]$ where the 1 is in the $i$ th position. Write $p_{\alpha}=\left[p_{\alpha}^{0}, \ldots, p_{\alpha}^{r}\right]$. Let $q_{i}=\left[p_{0}^{i}, \ldots, p_{s}^{i}\right] \in \mathbb{P}^{s}$ and let $w_{\alpha}=[0, \ldots, 0,1,0, \ldots, 0] \in \mathbb{P}^{s}$ where the 1 is in the $\alpha$ th position.

PROPOSITION 4.2. In the situation above, $\Gamma$ is associated to $\Gamma^{\prime}=\left\{w_{\alpha}, q_{i}\right\}$. 
Proof.

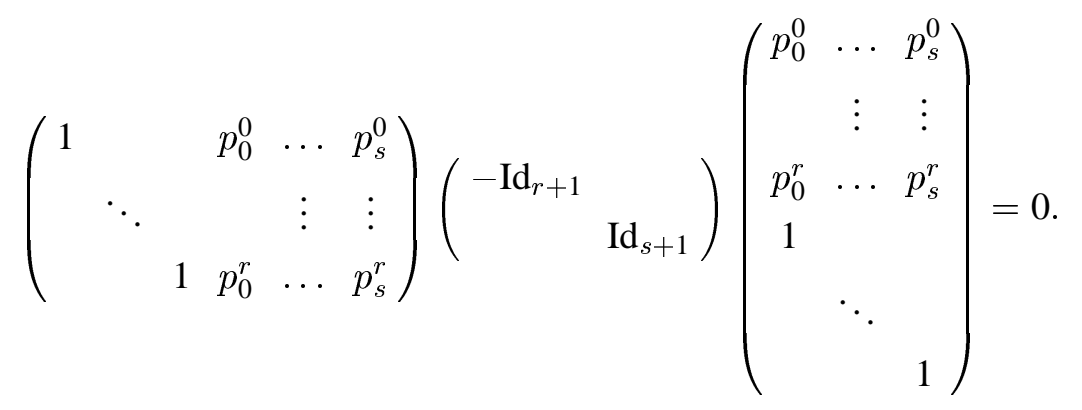

COROLLARY 4.3. $\operatorname{Span}\left\{p_{\alpha}\right\}=\mathbb{P}^{k}$ if and only if $\operatorname{Span}\left\{q_{i}\right\}=\mathbb{P}^{k}$.

Proof. Row rank equals column rank.

PROPOSITION 4.4 (Commutativity of association and Cremona). Let $z_{i}^{*}$ be the image simplex points determined by the standard Cremona transform of $\mathbb{P}^{r}$ in the $z_{i}$, which we denote $\phi_{z}$. Similarly, let $q_{\alpha}^{*}$ be the simplex points determined by the Cremona transform of $\mathbb{P}^{s}$ in the $q_{\alpha}$, which we denote $\phi_{q}$. Then the associated point set of $\Gamma^{*}=\left\{z_{i}^{*}, \phi_{z}\left(p_{\alpha}\right)\right\}$ is $\Gamma^{* *}=\left\{\phi_{q}\left(w_{\alpha}\right), q_{i}^{*}\right\}$, i.e., $\Gamma^{\prime *}=\Gamma^{* \prime}$.

Proof. The original set of points $z_{i}, p_{\alpha}$ yields a matrix

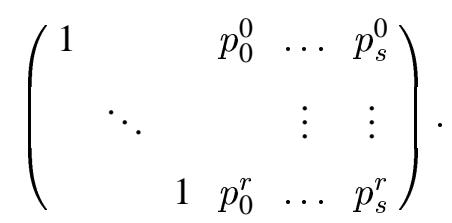

Under $\phi_{z}$ one gets a point set $z_{i}^{*}, \phi_{z}\left(p_{\alpha}\right)$ with matrix

$$
\left(\begin{array}{cccccc}
1 & & & \frac{1}{p_{0}^{0}} & \cdots & \frac{1}{p_{s}^{0}} \\
& \ddots & & & \vdots & \vdots \\
& & 1 & \frac{1}{p_{0}^{r}} & \cdots & \frac{1}{p_{s}^{r}}
\end{array}\right)
$$

whose association is explained above. But taking the transpose commutes with that of taking the Hadamard inverse.

DEFINITION 4.5. A set $\Gamma$ of $2 n+2$ points in $\mathbb{P}^{n}$ is said to be self-associated if it is associated to itself.

PROPOSITION 4.6. $\Gamma=\left\{z_{i}, p_{i}\right\}$ is self-associated if and only if the points sets $\left\{z_{i}\right\}$ and $\left\{p_{i}\right\}$ are both apolar with respect to some quadric $Q$. 
Proposition 4.6 generalizes the $n=2$ case which was known classically. A proof of the $n=2$ case is given in ([MSY], A.2.3).

Proof. Let $z_{i}$ be simplex points as above. We need to show the point set $\Gamma$ represented by the matrix ( $\mathrm{Id}, P$ ) is equivalent to the point set represented by the matrix $\left(\mathrm{Id},{ }^{t} P\right)$, where $P$ is a matrix whose columns are the entries of the $p_{i}$, if and only if the point sets are apolar with respect to a quadric $Q$. Let $Q$ also denote the $(r+1) \times(r+1)$ matrix representing the quadric. The point set $\Gamma^{\prime}$ consisting of the columns of $\left({ }^{t} P, \mathrm{Id}\right)$ is equivalent to the point set consisting of the columns of $\left({ }^{t} P Q, Q\right)$. The $p_{i}$ are $Q$-apolar if ${ }^{t} P Q=Q P^{-1}$, in which case $\left({ }^{t} P Q, Q\right)$ is equivalent to $\left(Q P^{-1}, Q\right)$ which is equivalent to $\left(P^{-1}, \mathrm{Id}\right)$ which is equivalent to (Id, $P$ ).

In light of (4.6), we can rephrase (1.8) yet again:

THEOREM 1.8 (Version 3). Let $\Gamma=\left\{z_{i}, p_{i}\right\} \subset \mathbb{P}^{n}$ be a self-associated point set. Then $\operatorname{Span}\left\{\phi_{z}\left(p_{i}\right)\right\} \neq \mathbb{P}^{2} \cdot \operatorname{Span}\left\{\phi_{z}\left(p_{i}\right)\right\}=\mathbb{P}^{1}$ if and only if $\Gamma$ is contained in a rational normal curve.

\section{Acknowledgements}

It is a pleasure to thank M. Kontsevich for suggesting the conjecture and useful comments, H. Pinkham for many useful conversations, S. Popescu for alerting the author to the relevant literature, interesting remarks, and useful comments, and the referee for useful comments and alerting the author to the reference [MSY].

\section{References}

[Con] Conner: Basic systems of rational norm-curves, American Journal of Mathematics 32 (1911), 115-176.

[DO] Dolgachev and Ortland: Point sets in projective spaces and theta functions, Astérisque 165 (1988), 210.

[EP1] Eisenbud, D. and Popescu, S.: Gale duality and free resolutions of ideals of points, preprint. [EP2] Eisenbud, D. and Popescu, S.: The projective geometry of the Gale transform, preprint.

[MSY] Matsumoto, K., Sasaki, T. and Yoshida, M.: The monodromy of the period map of a 4-parameter family of K3 surfaces and the hypergeometric function of type $(3,6)$, International Journal of Mathematics 3 (1992), 1-164. 